\title{
Spaces Matters: Classroom Acoustics and Repetitive Behaviors in Preschool Children with Autism
}

\author{
Shireen M. Kanakri \\ Department of Interior Design, Ball State University, Muncie, USA \\ Email address: \\ smkanakri@bsu.edu

\section{To cite this article:} \\ Shireen M. Kanakri. Spaces Matters: Classroom Acoustics and Repetitive Behaviors in Preschool Children with Autism. American Journal \\ of Pediatrics. Vol. 3, No. 6, 2017, pp. 89-94. doi: 10.11648/j.ajp.20170306.15
}

Received: September 28, 2017; Accepted: November 3, 2017; Published: November 30, 2017

\begin{abstract}
Autism has generally been ignored by the interior design community and excluded from building codes and guidelines, even those developed explicitly for special needs individuals. This research will look into how interior design factors affect individuals with autism; specifically with regards to acoustics. Today's world has put much emphasis and consideration towards the diversity of individuals and their developmental and psycho-social disorders, yet research has not been thorough in this topic; thus, this article presents a further step when considering development. Therefore, one of the primary aims of this research is to correct this exclusion by developing a preliminary framework of interior design guidelines for autism. To reach this goal of developing a framework for architectural guidelines for autism, an extensive literature review was conducted and a behavioral observation took place. Four classrooms were identified in two schools (two rooms in each school) based on their noise levels and behaviors were recorded from 42 participants. Research results indicate that environment is important to the treatment of autism because it influences behavior. A significant positive correlation between noise levels and frequency of target behaviors was found; that is, as decibel levels increased, several of the observed behaviors occurred with greater frequency. This research gives practical solutions that architects and designers can use to modify the environment for children with autism. Developed and expounded by the author in two previous studies (Kanakri, et al. "An Observational Study of Classroom Acoustical Design and Repetitive Behaviors in Children With Autism," Sage Publications, pp. 1-27, 2016) and (Kanakri, S. M., The Impact of Acoustical Environmental Design on Children with Autism. 2014), these analyses lay the groundwork for this article's research which provides tangible modifications to help these children develop their skills, cope with auditory problems and improve their behaviors.
\end{abstract}

Keywords: Autism, Interior Design, Noise, Behavior, Children, Classroom

\section{Introduction}

Autism spectrum disorder (ASD) has gained much attention as an important characteristic that affects the way individuals experience modern learning environments. ASD affects cognitive performances within a social, communicational, and behavioral area of functioning [1]. Though this disorder has become increasingly apparent among the general population, policy and design have struggled to implement evidencebased research into practical scenarios that are accommodating [2]. One particular difference and challenge for individuals with ASD is their increased capacity for auditory stimulation [3]. As a result, distress for these individuals is much higher and results in increased behaviors, adaptive and maladaptive $[4,5]$. If classrooms and learning environments are not designed to accommodate students with developmental disabilities, it can be assumed that they will not learn these important skills and may struggle to live in our society [21]. As the majority of individuals diagnosed with autism spectrum disorders (ASDs) live with sensory processing differences, the acoustic environment is of primary concern within interior design considerations [6]. A well-controlled environment with intentionally controlled sound levels can experimentally test the directional and temporal nature of this relationship [22]. This study looks at the way acoustic levels relate to behaviors based on observations of students with autism. This research paper explores how noise levels affect the behavior of children with autism in classroom settings and use the findings to generate future research ideas that will strengthen the empirical evidence for interior design guidelines for autism. 


\section{Background}

\subsection{Theories of Autism}

Autism spectrum symptoms have a number of theories used to help explain the phenomenon. The main idea is how differences in sensory inputs disrupt typical autonomous control and alter neural wiring [7]. Ultimately, it is agreed that the environment has a substantial role in the way individuals with autism learn and experience. Thus, the environmental conditions established by structural codes for learning environments requires reinterpretation with developing theoretical models of autism. For example, the Sensory Design Theory has much to offer the interior design community through its empirically backed evidence of environmental differences for learning between individuals [8].

\subsection{Noise and the Built Environment}

Understanding the role of sound within an environment, such as how differences in decibel level effect hearing ability, blood pressure, sleeping patterns, and stress levels, provides a necessary framework when designing environments for individual differences $[9,21]$.

\subsection{Autism and the Built Environment}

Appropriate interior design can help accommodate for individuals with autism. Methods such as way finding, sensory zoning, and adoption of escape spaces are unique options discussed among researchers as possible, and evidence based, ways to assist individuals with autism [10]. Though these ideas have promising implications, limited recommendations have been made within the regulatory system of designs for buildings.

\subsection{Acoustic Regulations for Learning Environment}

Previous research suggests that differences in perceptual processing to auditory, tactile, and visual stimuli can lead to individuals with autism being overwhelmed by sensory inputs, which further encumbers coordination and autonomous control, disrupting routes of prediction and anticipatory control, and over time altering neural wiring [7]. Therefore, persons with autism are particularly susceptible to negative effects of poorly designed acoustical environments. Hypersensitivity to sound has been discussed for decades as a very important concern within the ASD community. Between 1964 and 1994, the Autism Research Institute in California, USA, gathered the medical histories on more than 17,000 children with autism in different countries and more than $40 \%$ included parent notations of sound sensitivity [11]. The American National Standards Institute has an official statement for Performance Criteria, Design Requirements and Guidelines for Schools, which can be consulted for the acoustic design in schools [12]. The ANSI/ASA [12] has the following three standards for classroom acoustics; (1) unoccupied classroom levels must not exceed $35 \mathrm{~dB}$, (2) the signal-to-noise ratio should be at least $+15 \mathrm{~dB}$ at the child's ears, and (3) unoccupied classroom reverberation must not surpass $0.6 \mathrm{~s}$ in smaller classrooms or $0.7 \mathrm{~s}$ in larger rooms.

\subsection{Hypothesis}

It is hypothesized that there is a significant relationship between noise and the behavior of children with autism, with higher noise predicting an increase in repetitive behaviors in the classroom environment.

\section{Methods}

The behavioral observations took place in four classrooms, two classrooms from each of the two schools. These classrooms were separated into quiet and noisy according to $\mathrm{dB}$ guidelines defined by WHO and ANSI. Classrooms with an average of $35 \mathrm{~dB}$ or greater while unoccupied were considered noisy, and those with an average of less than $35 \mathrm{~dB}$ while unoccupied were considered quiet. Each classroom had similar acoustical and lighting design elements, but the decibel levels varied based on their proximity to louder areas such as playgrounds, multipurpose rooms, and rooms containing HVAC systems. Participants consisted of children with high functioning autism in the second and third grades at two schools for autism. Two classrooms of children from each school participated in the study.

A 12-hour pilot study was conducted to test the hypothesized behaviors to be observed. Five behaviors were compiled from an extensive literature review on the impacts of noise on behaviors in individuals with autism. The pilot study tested the presence of the list of behaviors compiled from literature. After the 12 hours of observation in the pilot study, two additional behaviors were deemed important to track. In addition to the original five behaviors, covering ears and repetitive speech were added as essential items to count during observation. In total, seven behaviors were tracked in this study. While some of these behaviors can be readily associated with the Restricted and Repetitive Behaviors Questionnaire [4, 5], the present list was compiled to directly address behaviors in the classroom that can be observed in a specified amount of time, and that would be more directly associated with auditory distress [13]. These behaviors consisted of the following:

1. Repetitive movement (stereotypy): defined as a type of repetitive movement with the hands, legs or any part of the body, as when, for example, the child flaps his hands many times continuously [14]. Other examples of a repetitive movement would include rocking, twirling, and spinning.

2. Repetitive speech: This behavior is related to any type of repetitive and continuous speech. Repetitive speech patterns are a frequently characteristic of children in the autism spectrum [15]. Words may be repeated over and over (echolalia), and words or phrases previously heard may be repeated after a time delay of a few minutes, hours, days, weeks, or even months, in the case of 
delayed echolalia [14].

3. Covering the ears: Children may cover both ears and others cover one ear [16]. While this behavior is functional for ear protection, it is identified as intrusive when children must persistently keep their ears covered and may be linked to anxiety as a child becomes fearful of potential unpleasant noises, and pre-emptively engages in ear-covering.

4. Hitting response: This response is related to any type of hitting, including children hitting themselves, other children, or throwing objects which can injure themselves or others. Turner [17] defined the hit response as any behavior that can cause tissue damage (bruises, redness, and open wounds). Common forms include head banging, hand biting, and excessive scratching or rubbing.

5. Produce loud sounds: Continuous loud noises are produced during any kind of activity. These noises could be clear words, unclear words, or production of loud sounds.

6. Blinking eyes: Eye blinking is an involuntary process that helps keep the eyes hydrated and protected [18]. Eye blinking beyond a normal level of 44 minutes per day provides a way to measure social disengagement [18]. The more the child blinks, the more he is disengaged in the event [19].

7. Complaining: Complaining occurs when the children refuse to do what the teacher is asking them to do, expressing disinterest or direct refusal to follow directions, or complaints about being bothered by something in the classroom environment.

Forty two total students participated in this study (20 from School 1 and the remaining 22 from School 2). All students had been diagnosed with high-functioning autism but there was a range in different intellectual abilities within each classroom. Please see Figure 1 and Figure 2.
Observations of the students were focused on 7 behaviors typical of a child with autism at this age: repetitive movement, repetitive speech, covering the ears, hitting, loud sounds, blinking eyes, and complaining. The observation period lasted 7 weeks, with 20 total hours of observation being completed in each classroom. Three separate techniques of observation were used. First, the researcher used the Noldus Observer XT software to determine the number of times each of the 7 problem behaviors occurred. Second, every 10 seconds, a video recorder connected to a decibel meter recorded the children's behavior. Lastly, a teacher questionnaire was utilized.

\section{Results}

When the data for all four classrooms were combined, a significant positive correlation was found between the decibel levels and complaining, repetitive speech, hitting, producing loud sound, repetitive motor movement, blinking eyes and covering ears. As the levels of decibel increased, the occurrence of these behaviors also increased. There were also differences in behaviors expressed depending the classification of the classroom into either quiet or loud. The loud classrooms had significantly more observances of complaining, repetitive speech, producing loud sounds, repetitive motor movement, and covering ears than the quiet classrooms. In general, complaining, repetitive speech, hitting, production of loud sounds, and covering ears occurred more frequently when noise levels were greater than $70 \mathrm{~dB}$, in comparison with when decibel was less than $55 \mathrm{~dB}$ or between 55 and $70 \mathrm{~dB}$. The quiet classrooms had significantly more observances of covering ears than the loud classroom, see Table 1 . This research builds upon previously known phenomenon regarding the relationship between repetitive behaviors and auditory processing [11, 20,21].

Table 1. Behavior means and standard deviations with t-test information.

\begin{tabular}{|c|c|c|c|c|c|c|c|c|c|}
\hline \multirow{2}{*}{$\begin{array}{l}\text { Classroom } \\
\text { Behavior }\end{array}$} & \multicolumn{3}{|c|}{ School I $(N=268)$} & \multicolumn{3}{|l|}{ School II (N = 222) } & \multicolumn{3}{|c|}{ Both Schools $(N=490)$} \\
\hline & t-value (df) & $\begin{array}{l}\text { Quiet Mean } \\
\text { (SD) }\end{array}$ & $\begin{array}{l}\text { Loud Mean } \\
\text { (SD) }\end{array}$ & t-value (df) & $\begin{array}{l}\text { Quiet Mean } \\
\text { (SD) }\end{array}$ & $\begin{array}{l}\text { Loud Mean } \\
\text { (SD) }\end{array}$ & t-value (df) & $\begin{array}{l}\text { Quiet Mean } \\
(S D)\end{array}$ & $\begin{array}{l}\text { Loud Mean } \\
(S D)\end{array}$ \\
\hline Complain & $1.73(144.50$ & $1.05(2.77)$ & $2.90(12.00)$ & $3.29(121.98)^{* *}$ & $1.00(2.28)$ & $2.85(5.12)$ & $2.80(252.69)$ & $1.03(2.54)$ & $2.89(9.72)$ \\
\hline $\begin{array}{l}\text { Repetitive } \\
\text { Speech }\end{array}$ & $4.85(223.22)^{* * *}$ & $16.29(21.39)$ & $32.86(33.08)$ & $6.42(160.44)^{* * *}$ & $2.74(6.81)$ & $10.19(9.66)$ & $6.30(364.95)^{* * *}$ & $9.75(17.44)$ & $23.37(28.26)$ \\
\hline Hit & $0.53(266)$ & $0.85(3.62)$ & $1.12(4.41)$ & $3.54(106.10)^{* *}$ & $0.25(0.64)$ & $1.07(2.19)$ & $1.83(407.18)$ & $0.56(2.66)$ & $1.09(3.64)$ \\
\hline Blink Eyes & $1.47(220.46)$ & $1.29(3.81)$ & $2.20(6.02)$ & $-1.92(203.83)$ & $2.62(5.78)$ & $1.45(3.18)$ & $-0.101(488.00)$ & $1.94(4.90)$ & $1.89(5.03)$ \\
\hline $\begin{array}{l}\text { Produce Loud } \\
\text { Sound }\end{array}$ & $-0.85(255.68)$ & $10.92(15.52)$ & $9.46(12.28)$ & $9.22(116.42)^{* * *}$ & $4.04(6.98)$ & $21.57(17.53)$ & $5.30(430.17)^{* * *}$ & $7.60(12.62)$ & $14.53(15.84)$ \\
\hline $\begin{array}{l}\text { Repetitive } \\
\text { Motor } \\
\text { Movement }\end{array}$ & $1.15(266.00)$ & $16.39(24.40)$ & $19.52(20.04)$ & $4.32(150.95)^{* * *}$ & $19.73(21.16)$ & $36.34(32.73)$ & $3.72(442.71)^{* * *}$ & $18.00(22.91$ & $26.56(27.34)$ \\
\hline Cover Ears & $5.05(189.56)^{* * *}$ & $6.38(15.53)$ & $21.80(31.60)$ & $-3.94(207.04)^{* * * *}$ & $6.24(10.08)$ & $2.02(5.75)$ & $3.75(321.77)^{* * *}$ & $6.32(13.16)$ & $13.52(26.23$ \\
\hline
\end{tabular}

${ }^{1}$ Reprinted from "An Observational Study of Classroom Acoustical Design and Repetitive Behaviors in Children with Autism,” Kanakri, S. M., et al, 2016, Sage Publications, p. 18 


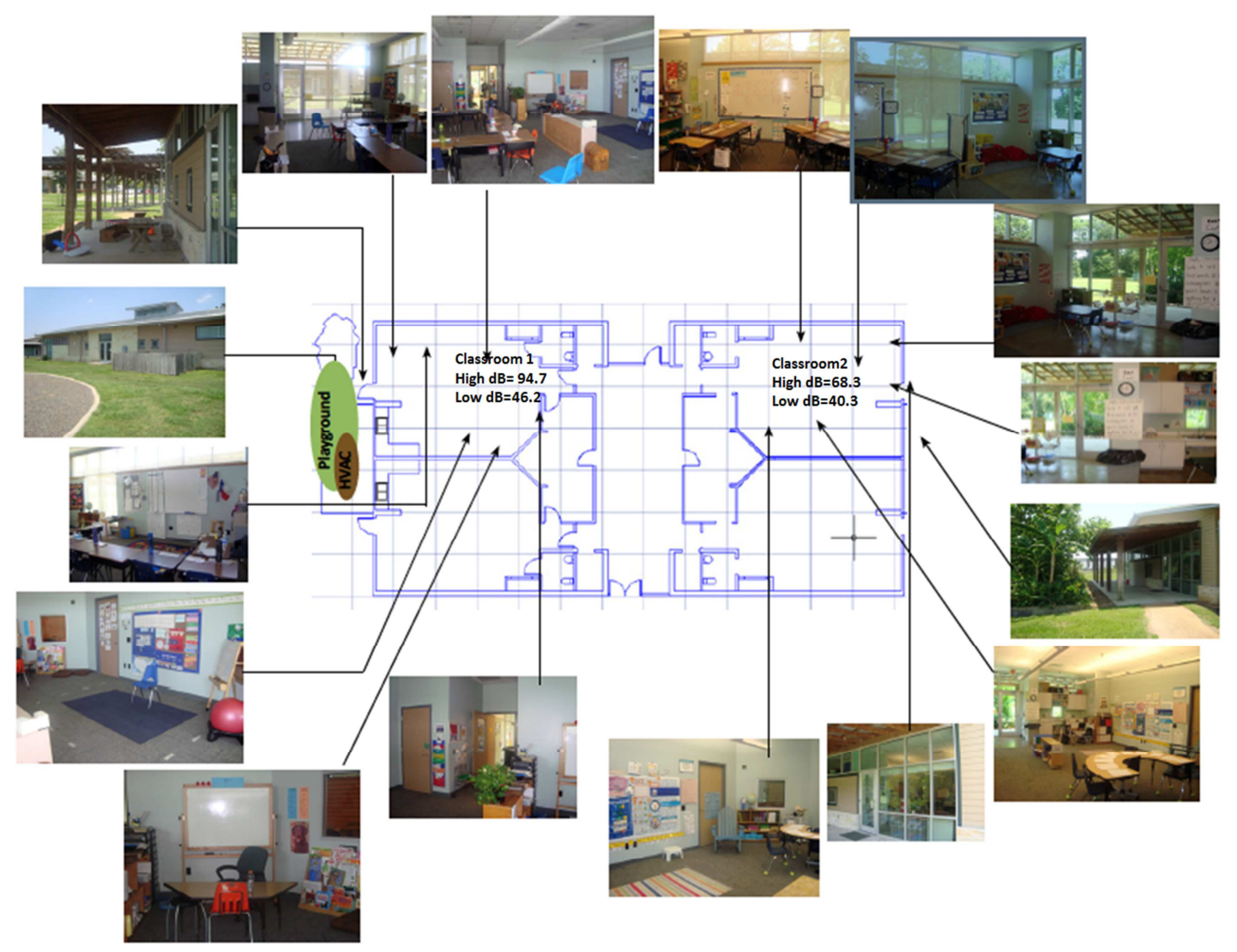

Figure 1. School 1 layout and images. ${ }^{2}$

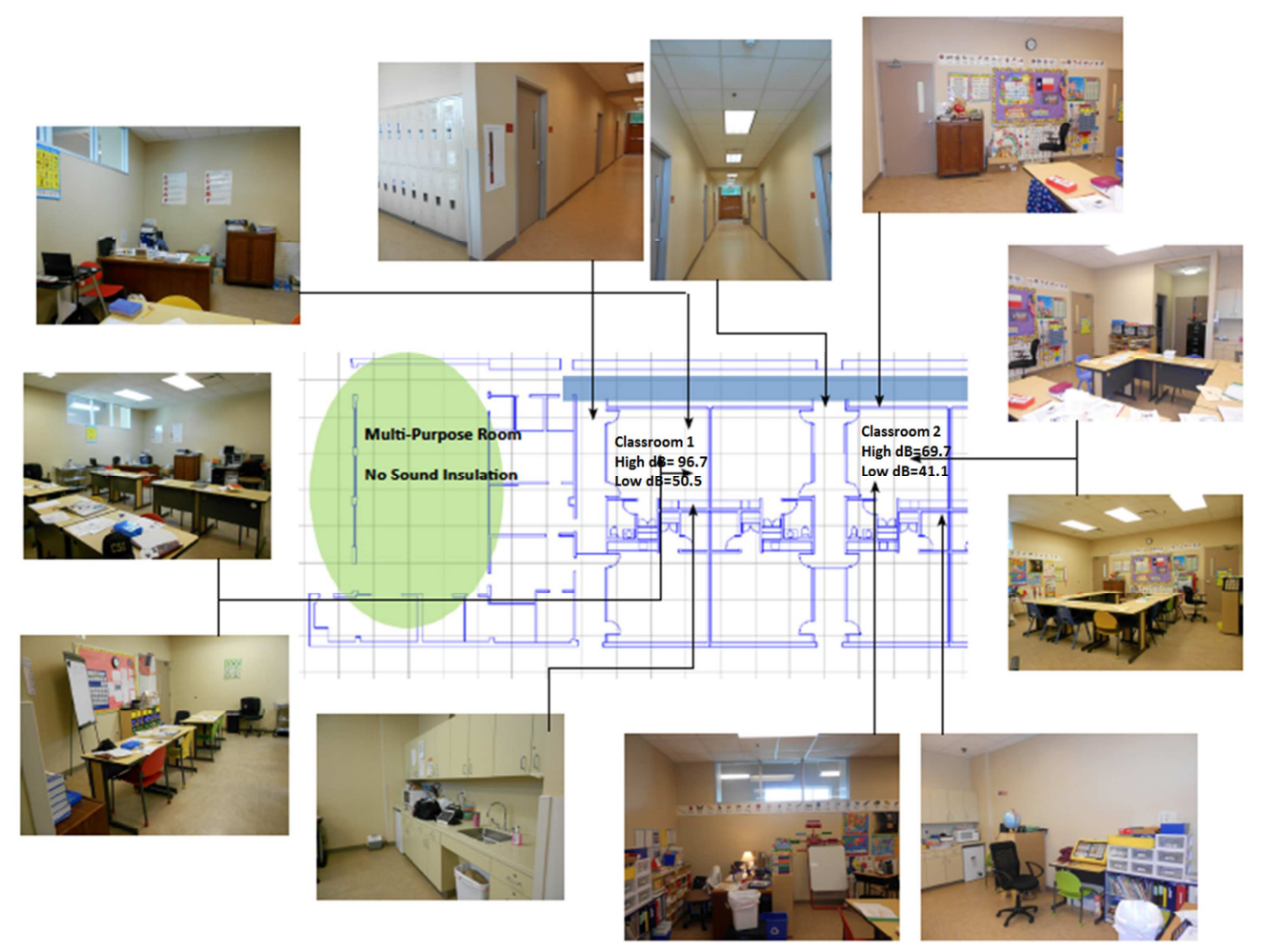

Figure 2. School 2 layout and images. ${ }^{3}$

${ }^{2}$ Reprinted from "The Impact of Acoustical Environmental Design on Children with Autism," Kanakri, S. M., 2014, p. 40

${ }^{3}$ Reprinted from "The Impact of Acoustical Environmental Design on Children with Autism," Kanakri, S. M., 2014, p. 41. 


\section{Discussion}

In general, our results show a significant positive correlation between decibel levels and most behaviors observed in this study. These findings offer confirmation that a noisy acoustical environment and repetitive behaviors have a direct relationship. As a strength, this research shows a distribution of behaviors and how each one may be utilized based on the acoustic environment. Understanding the frequency of behavior requires an in-depth look at the many elements present in an environment, and acoustics are conceptually concrete and practical for building specifications [10]. As a weakness, the decibel variable is organized based on decibel groups such as less than $55 \mathrm{~dB}$, $55-70 \mathrm{~dB}$, and 70 or higher $\mathrm{dB}$; future research should control for these sounds more precisely in order to obtain stronger empirical support for correlational support with studied behaviors. Specifically, there was a difference in decibel levels between the two schools under observation, which emphasizes the need for more constant decibel levels when observing. It is important that future research test the causal association between these two factors in an even-more precise manner. Classroom environments with intentionally controlled sound levels can be experimentally tested for the directional and temporal nature of this relationship.

\section{Conclusion}

Overall, findings suggest that attention to acoustic design and modifications to existing environments are essential to providing a supportive educational environment. Although under some circumstances, individuals with autism may benefit from being acclimated to neuro-typical environments, providing environments that buffer acoustic benefits the learning of individuals with heightened sensory perception and individuals with neuro-typical functioning alike. Specifically, we theorize that with decreased environmental stimuli, attentional processes and executive attentional resources can be relocated toward individual processing. With decreased arousal and increased neurological resources, individuals with ASD will evidence less stereotypic behavior, as a result of lower internal distress. Future research might address this directly through taking physiological measurements or ratings of children's subjective feelings periodically throughout the day. Future studies should address questions regarding the causality of these behaviors within specific acoustic settings and what changes to interior design become the most beneficial for individuals with autism to produce the highest level of efficiency for design.

\section{Acknowledgements}

Data reported in this paper are taken directly from the author's dissertation: Kanakri, S. M., The Impact of Acoustical Environmental Design on Children with Autism (2014).

\section{References}

[1] Diagnostic and statistical manual of mental disorders: DSM-5. Washington, Londres: American Psychiatric Association, 2013.

[2] H. E. Dingfelder and D. S. Mandell, "Bridging the Researchto-Practice Gap in Autism Intervention: An Application of Diffusion of Innovation Theory," Journal of Autism and Developmental Disorders, vol. 41, no. 5, pp. 597-609, 2010.

[3] A. Remington and J. Fairnie, "A sound advantage: Increased auditory capacity in autism," Cognition, vol. 166, pp. 459465, 2017.

[4] S. R. Leekam, M. R. Prior, and M. Uljarevic, "Restricted and repetitive behaviors in autism spectrum disorders: A review of research in the last decade.," Psychological Bulletin, vol. 137, no. 4, pp. 562-593, 2011.

[5] S. Leekam, J. Tandos, H. Mcconachie, E. Meins, K. Parkinson, C. Wright, M. Turner, B. Arnott, L. Vittorini, and A. L. Couteur, "Repetitive behaviours in typically developing 2-year-olds," Journal of Child Psychology and Psychiatry, vol. 48, no. 11, pp. 1131-1138, 2007.

[6] C. S. Martin, "Exploring the impact of the design of the physical classroom environment on young children with autism spectrum disorder (ASD)," Journal of Research in Special Educational Needs, vol. 16, no. 4, pp. 280-298, 2014.

[7] M. Brincker and E. B. Torres, "Noise from the periphery in autism," Frontiers in Integrative Neuroscience, vol. 7, no. 34, 2013.

[8] M. Mostafa, "Architecture for autism: Autism ASPECTSS in school design," International Journal of Architectural Research, vol. 8, pp. 143-158, 2014.

[9] E. Atmaca, I. Peker, and A. Altin, "Industrial noise and its effects on humans.," Polish Journal of Environmental Studies, vol. 14, pp. 721-726, 2005.

[10] K. Mcallister and B. Maguire, "Design considerations for the autism spectrum disorder-friendly Key Stage 1 classroom," Support for Learning, vol. 27, no. 3, pp. 103-112, 2012.

[11] B. Rimland and S. M. Edelson, "Brief report: A pilot study of auditory integration training in autism," Journal of Autism and Developmental Disorders, vol. 25, no. 1, pp. 61-70, 1995.

[12] American National Standards Institute/Acoustical Society of America. "American National Standard Acoustical Performance Criteria, Design Requirements, and Guidelines for Schools, Part 1: Permanent Schools.” ANSI/ ASA S12.602010/Part 1, 2012.

[13] M. Kinnealey, B. Pfeiffer, J. Miller, C. Roan, R. Shoener, and M. L. Ellner, "Effect of Classroom Modification on Attention and Engagement of Students With Autism or Dyspraxia," American Journal of Occupational Therapy, vol. 66, no. 5, pp. 511-519, 2012 .

[14] T. Insel, "Post by Former NIMH Director Thomas Insel: Transforming Diagnosis," National Institute of Mental Health, 29-Apr-2013. [Online]. Available: https://www.nimh.nih.gov/about/directors/thomasinsel/blog/2013/transforming-diagnosis.shtml. [Accessed: 27Sep-2017]. 
[15] O. I. Lovaas, J. W. Varni, R. L. Koegel, and N. Lorsch, "Some Observations on the Nonextinguishability of Childrens Speech," Child Development, vol. 48, no. 3, p. 1121, 1977.

[16] J.-C. Tang, C. H. Kennedy, A. Koppekin, and M. Caruso, "Functional analysis of stereotypical ear covering in a child with autism.," Journal of Applied Behavior Analysis, vol. 35, no. 1, pp. 95-98, 2002.

[17] M. Turner, "Annotation: Repetitive Behaviour in Autism: A Review of Psychological Research," Journal of Child Psychology and Psychiatry, vol. 40, no. 6, pp. 839-849, 1999.

[18] J. Goodwin, Blink patterns may be a window into autistic mind: Watching a video, toddlers with autism reacted to action, not emotional content. 2011.
[19] S. Shultz, A. Klin, and W. Jones, "Inhibition of eye blinking reveals subjective perceptions of stimulus salience," Proceedings of the National Academy of Sciences, vol. 108, no. 52, pp. 21270-21275, Dec. 2011.

[20] R. R. Grinker, Unstrange minds: Remapping the world of autism. New York, NY: Basic Books, 2007.

[21] S. M. Kanakri, The Impact of Acoustical Environmental Design on Children with Autism. 2014.

[22] S. M. Kanakri, M. Shepley, L. G. Tassinary, J. W. Varni, and H. M. Fawaz. An Observational Study of Classroom Acoustical Design and Repetitive Behaviors in Children With Autism. Sage Publications, pp. 1-27, 2016. 